\title{
A Generalization of Dehn-Sommerville Relations to Simple Stratified Spaces*
}

\author{
Ketan Mulmuley \\ Department of Computer Science, University of Chicago, \\ Chicago, IL 60637, USA
}

\begin{abstract}
We generalize the classical Dehn-Sommerville relations for simple, convex polytopes to compact, transverse intersections of arbitrary smooth manifolds with boundaries.
\end{abstract}

\section{Introduction}

Let $P$ be a $d$-dimensional, convex polytope and let $f^{i}(P)$ denote the number of its $i$-faces, where $i<d$. By convention, $f_{-1}(P)=1$.

The well-known Euler's relation for convex polytopes states that

$$
\sum_{i=-1}^{d-1}(-1)^{i} f^{i}(P)=(-1)^{d-1}
$$

Dehn-Sommerville relations [2], [9] generalize Euler's relation further, in case $P$ is simplical, i.e., if every face of $P$ is a simplex: For $-1 \leq k \leq d-2$,

$$
\sum_{j=k}^{d-1}(-1)^{j}\left(\begin{array}{l}
j+1 \\
k+1
\end{array}\right) f^{j}(P)=(-1)^{d-1} f^{k}(P) .
$$

If $k=-1$, we recover Euler's relation.

In addition to boundary complexes of simplical polytopes, these relations also hold for triangulations of topological and homology $(d-1)$-spheres and Klee's Eulerian $(d-1)$-spheres [6]. See [5] for a historical survey.

\footnotetext{
* This research was supported by NSF Grant CCR 8906799 and a Packard fellowship.
} 
A dual of a simplicial convex polytope is a simple polytope. More precisely, let $P$ be a bounded, convex polytope in $R^{d}$ bounded by $n$ half-spaces given by $g_{i}(x)=g_{i}\left(x_{1}, \ldots, x_{d}\right) \leq 0$, where $g_{i}$ denotes a linear functional. We say that $P$ is simple if every 0 -face (vertex) of $P$ is contained in precisely $d$ of the bounding hyperplanes. Note that we are assuming that $P$ is bounded. Let $f^{i}(P)$ denote the number of $i$-faces of $P$, where $0 \leq i \leq d$. The interior of $P$ is the unique $d$-dimensional face of $P$ and hence $f^{d}(P)=1$.

For a simple polytope $P$, relations (1) can be rewritten as follows:

\section{Dehn-Sommerville Relations.}

$$
\sum_{j=0}^{i}(-1)^{j}\left(\begin{array}{l}
d-j \\
d-i
\end{array}\right) f^{j}(P)=f^{i}(P), \quad 0 \leq i \leq d .
$$

Note that Dehn-Sommerville relations apply only when $P$ is simple. However, if $P$ is not simple, almost every infinitesimal perturbation of $P$ is simple. More precisely,

Fact 1.1. For almost all, arbitrarily small, $\varepsilon_{i}, P^{\prime}=\bigcap\left\{g_{i}(x) \leq \varepsilon_{i}\right\}$ is simple.

One natural question that arises is whether it is possible to extend DehnSommerville relations to semi-algebraic sets of the form $\bigcap\left\{g_{i}(x) \leq\right.$ (or $\left.\left.=\right) 0\right\}$, where $g_{i}(x)=g_{i}\left(x_{1}, \ldots, x_{d}\right)$ are now polynomials in $x_{1}, \ldots, x_{d}$. In this paper we answer this question in the affirmative.

Just like the classical Dehn-Sommerville relations applied only to simple, bounded, convex polytopes, the extended relations can be expected to apply to the sets of the form $\bigcap\left\{g_{i}(x) \leq\right.$ (or $\left.\left.=\right) 0\right\}$, only if they are compact, and simple. The exact definition of simplicity in the nonlinear setting is given in Section 3 . Here we merely note that the following analogue of Fact 1.1 holds.

Theorem 1.1. For almost all, arbitrarily small, $\varepsilon_{i}, Z^{\prime}=\bigcap\left\{g_{i}(x) \leq(\right.$ or $\left.=) \varepsilon_{i}\right\}$ is simple.

This theorem is proved in Section 3 using transversality theory for manifolds.

Now let $Z=\bigcap\left\{g_{i}(x) \leq\right.$ (or $\left.\left.=\right) 0\right\}$ be a compact, simple set in $R^{d}$. Then $Z$ admits a natural Whitney stratification, i.e., a partition into disjoint, open manifolds, called strata, of dimension $\leq \operatorname{dim}(Z)$. For a stratum $S$ of $Z$, let $\chi(S)$ denote its Euler's characteristic. Let

$$
f^{i}(Z)=\sum_{S} \chi(S)
$$

where $S$ ranges over all $i$-dimensional strata of $Z$. Then

Theorem 1.2. For $0 \leq i \leq c=\operatorname{dim}(Z)$,

$$
\sum_{j=0}^{i}(-1)^{j}\left(\begin{array}{l}
d-j \\
d-i
\end{array}\right) f^{j}(Z)=f^{i}(Z) .
$$


Note that when $g_{i}(x)$ are linear functionals, then $Z$ is a simple convex polytope. Its strata are its faces, and Euler's characteristic of any of its faces is 1 . Thus in this case $f^{i}(Z)$ is simply the number of $i$-dimensional faces of $Z$ and we recover the classical Dehn-Sommerville relations.

A principle tool in the proof of Theorem 1.2 is the following duality theorem for manifolds [7].

Lefchetz Duality. Let $(X, A)$ be a compact, relative d-manifold. This means $X$ is compact, $A \subseteq X$, and the complement $X \backslash A$ is a d-manifold. Let $\mathbf{F}$ be a fixed field. If the pair $(X, A)$ is orientable, then the following isomorphisms hold:

$$
H_{i}(X, A ; \mathbf{F}) \cong H^{d-i}(X \backslash A ; \mathbf{F}), \quad 0 \leq i \leq d,
$$

where $H_{i}(X, A ; \mathbf{F})$ denotes the ith relative, singular homology group of the pair $(X, A)$ over $\mathbf{F}$ and $H^{j}(X \backslash A ; \mathbf{F})$ denotes the $j$ th singular cohomology group of $X \backslash A$ over $\mathbf{F}$. If $(X, A)$ is not orientable, then the same isomorphisms hold if we let $\mathbf{F}=\mathbf{F}_{2}$.

That duality on manifolds should turn out to be crucial in the proof of Theorem 1.2 is not unexpected. In fact, the classical Dehn-Sommerville relations for a simple, convex polytope $P$ can be rewritten in the following transparent form:

$$
h^{i}(P)=h^{d-i}(P), \quad 0 \leq i \leq d,
$$

where $h^{i}(P)$ are entries of the so-called $h$-vector [8]. They are defined as follows:

$$
h^{i}(P)=\sum_{j=0}^{d}(-1)^{i+j}\left(\begin{array}{l}
j \\
i
\end{array}\right) f^{j}(P), \quad 0 \leq i \leq d
$$

The relations (3) have some resemblance with the statement of Lefschetz duality. It is a remarkable result of Stanley [10], that this resemblance runs deep. For every simplical, convex polytope $P$, he constructs a toric variety $X(P)$, whose ith Betti number is precisely $h^{i}(P)$. Then Dehn-Sommerville relations for $P$ are implied by Poincare Duality for $X(P)$. Stanley's construction, however, works only if $P$ is a simplical, convex polytope. In particular, it is not applicable to arbitrary triangulation of a $d$-sphere. (There are triangulations of $d$-spheres, the so-called "nonshellable" triangulations, which are not homeomorphic to the boundary complex of a convex polytope.) In the light of our generalization, it is of interest to investigate under what conditions can one construct a variety $X(Z)$ corresponding to a given simple set $Z=\bigcap\left\{g_{i}(x) \leq\right.$ (or $\left.\left.=\right) 0\right\}$, so that the $i$ th Betti number of $X(Z)$ is precisely $h^{i}(Z)$, as defined above, where $f^{i}(Z)$ is now defined as in (2).

Finally, let us remark that Theorem 1.2 applies not just to simple sets of the form $\bigcap\left\{g_{i}(x) \leq\right.$ (or $\left.\left.=\right) 0\right\}$, but to any compact, simple, stratified space. For example, the intersection $\bigcap_{i=1}^{n} M_{i}$ of $n$ smooth manifolds $M_{i}$ with boundaries is simple if the manifolds and their boundaries intersect transversally; see Section 3.

The outline of the rest of the paper is as follows. In Section 2 we recall 
some basic results regarding Whitney stratification and transversality theory of manifolds. In Section 3 we prove Theorem 1.1 and 1.2.

\section{Preliminaries}

Let $X, Y$ be smooth manifolds and let $Z \subseteq Y$ be a smooth submanifold. A smooth map $f: X \rightarrow Y$ is called transverse to $Z$ if, for all $x \in X$, such that $f(x) \in Z$, $d f(x) T_{x} X+T_{f(x)} Z=T_{f(x)} Y$. Here $d f(x)$ denotes the derivative of $f$ at $x, T_{x} X$ denotes the tangent space of $X$ at $x . T_{f(x)} Z$ and $T_{f(x)} Y$ respectively denote tangent spaces of $Z$ and $Y$ at $f(x)$. An important result in the transversality theory of manifolds is the following [3].

Transversality Theorem. Let $X, B, Y$ be smooth manifolds and let $Z \subseteq Y$ be a submanifold. If the smooth map $g: X \rightarrow Y$ is transverse to $Z$, then for almost all values $b \in B, g_{b}: X \rightarrow Y$, given by $g_{b}(x)=g(x, b)$, is transverse to $Z$.

Now let us recall a few results regarding Whitney stratification, see [4]. Let $M$ be a smooth manifold and let $Z \subseteq M$ be a $d$-dimensional subset of $M$ that admits Whitney stratification. Roughly this means that $Z$ can be partitioned into a finite collection of disjoint, open manifolds, called strata, of dimension $\leq d$, which are "glued" together in a nice fashion. If $Z_{1} \subseteq M$ and $Z_{2} \subseteq M_{2}$ are Whitney stratified subsets of smooth manifolds $M_{1}$ and $M_{2}$ and if $g: M_{1} \rightarrow M_{2}$ is a smooth map, we say that the restriction $f \mid Z_{1}: Z_{1} \rightarrow M_{2}$ is transverse to $Z_{2}$ if, for each stratum $A$ in $Z_{1}$, and each stratum $B$ in $Z_{2}$, the map $f \mid A \rightarrow M_{2}$ is transverse to $B$. A special case arises when $M_{1}=M_{2}$ and $g$ is the identity map. In this case we say that $Z_{1}$ and $Z_{2}$ are transversal.

The following are some basic results regarding Whitney stratifications.

1. If $Z \subseteq M_{2}$ is a Whitney stratified subspace of the manifold $M_{2}$ and if $g: M_{1} \rightarrow M_{2}$ is a smooth map that is transverse to $Z$, then $g^{-1}(Z)$ is a Whitney stratified subspace of $M_{1}$ with a canonical stratification, obtained by pulling back the Whitney stratification of $Z$ through $g$.

2. If $Z_{1}, Z_{2} \subseteq M$ are two Whitney stratified subspaces of $M$, then $Z_{1} \cap Z_{2}$ is also a Whitney stratified space whose strata are the intersections of the strata of $Z_{1}$ and $Z_{2}$.

A key construction that we need on a Whitney stratification is the so-called normal slice construction [4]. Let $Z \subseteq M$ be a Whitney stratified subspace of $M$. Let $p \in Z$ be a point and let $S(p)$ be the stratum of $Z$ containing $p$. Let $D(p)$ be a very small, smooth disk in $M$, that is transverse to $Z$, such that $D(p) \cap S(p)=p$. The dimension of $D(p)$ will necessarily be the dimension of $M$ minus the dimension of $S$. Then $N(P)=D(p) \cap Z$ is called the normal slice at $p$. Because it is the intersection of Whitney stratified spaces, it has a canonical stratification obtained by intersecting the strata of $D(p)$ and $Z$. If $S(p)$ is a connected stratum, then the topological type of $N(p)$ depends only on the stratum $S(p)$. This is essentially a consequence of Thom's Isotopy Lemma; see [4]. 


\section{Simple Stratified Spaces}

Every $d$-simplex $S \subseteq R^{d}$ has a natural stratification, in which each $j$-face of $S$ constitutes a distinct stratum. This is not the only stratification of $S$. For example, we can consider two different $j$-faces of $S$ as constituting a single stratum, because a stratum does not have to be connected. In what follows, we are only concerned with the natural stratification of a simplex.

Definition. A Whitney stratified space $Z \subseteq M$ is called simple if, for each point $p \in Z$, the normal slice $N(p)$ is homeomorphic to a $d$-ball, where $d=\operatorname{dim}(Z)-$ $\operatorname{dim}(S(p))$, and the resulting stratification of $N(p)$ coincides with the natural stratification of a $d$-simplex.

A simple, convex polytope $P$ is obviously a simple, stratified space, where each $j$-face of $P$ constitutes a stratum. Another example of a simple, stratified space is a smooth manifold $M$ with boundary $\partial M$. Here $\{M, M \backslash \partial M\}$ gives a natural, simple stratification of $M$. A normal slice at any point on $\partial M$ is a 1 -simplex and that at any point in $M \backslash \partial M$ is a 0 -simplex.

If we consider a solid torus $M$ with a meridian circle $C$ on its boundary, then we do not get a simple stratification. To see this let $p \in C$. Then $N(p)$ is definitely a 2-simplex. However, in the resulting stratification of $N(p)$ the two 1-faces that are adjacent to $p$ constitute a single stratum, because they result from the single (unique) two-dimensional stratum of $M$. Thus $N(p)$ does not have the natural stratification of a 2 -simplex.

The set of simple, stratified spaces is quite rich, as the following results will show.

Theorem 3.1. Let $Z \subseteq M_{2}$ be a simple, stratified space. Let $g: M_{1} \rightarrow M_{2}$ be a smooth map that is transverse to $Z$. Then $f^{-1}(Z)$ is also a simple stratified space.

Proof. We have already seen that $f^{-1}(Z)$ is a Whitney stratified space, with stratification that is obtained by pulling back the stratification of $Z$ through $f$. Let $p \in f^{-1}(Z)$ be a point. Let $S(p)$ be the stratum of $f^{-1} Z$ containing $p$. Let $S(f(p))$ be the stratum of $Z$ containing $f(p)$. Let $N(f(p))$ be a normal slice at $f(p)$ that is transverse to $f$ (we can always choose $N(f(P))$ that is transverse to $f$ ). Then it is clear, because of transversality of $f$, that $f^{-1}(N(f(p)))$ is a normal slice of $f^{-1}(Z)$ at $p$. Moreover, transversality of $f$ also implies that both $f^{-1}(N(f(p)))$ and $N(f(p))$ have the same topological type. Because $Z$ is simple, $N(f(p))$ has the natural stratification of a simplex, with dimension $=\operatorname{dim}(Z)-\operatorname{dim}(S(f(p)))$. It follows that $f^{-1}(N(f(p)))$, the normal slice at $p$, has the stratification of a simplex with dimension $\operatorname{dim}\left(f^{-1}(Z)-\operatorname{dim}(S(p))=\operatorname{dim}(Z)-\operatorname{dim}(S(f(p)))\right.$; the latter equality follows from transversality.

Corollary 3.1. If $g_{i}(x)=g_{i}\left(x_{1}, \ldots, x_{d}\right), i \leq n$, are arbitrary polynomials, then for almost all, arbitrarily small, $\varepsilon=\left(\varepsilon_{1}, \ldots, \varepsilon_{n}\right), Z=\bigcap\left\{g_{i}(x) \leq(o r=) \varepsilon_{i}\right\}$ is a simple, stratified space. 
Proof. Let $y_{1}, \ldots, y_{n}$ be a coordinate system in $R^{n}$. Then $Y=\bigcap\left\{y_{i} \leq\right.$ (or $\left.\left.=\right) 0\right\}$ is a simple, stratified space with a natural stratification. Moreover, $Z=f^{-1}(Y)$, where $f: R^{\mathrm{d}} \rightarrow R^{n}$ is given by $f(x)=\left(g_{1}(x)-\varepsilon_{1}, \ldots, g_{n}(x)-\varepsilon_{n}\right)$. We will be done, by Theorem 3.1, if we can show that $f$ is transverse to $Y$. However, $g: R^{d} \times R^{n} \rightarrow R^{n}$, given by $g(x, \varepsilon)=\left(g_{1}(x)-\varepsilon_{1}, \ldots, g_{n}(x)-\varepsilon_{n}\right)$, is obviously transverse to $Y$, it being a submersion. As $f(x)=g(x, \varepsilon)$ we are done by applying the Transversality Theorem to $g$.

Theorem 3.2. Let $Z_{1}, Z_{2} \leq M$ be two simple, stratified spaces that are transverse to each other. Then $Z_{1} \cap Z_{2}$ is also a simple stratified space.

Proof. We know that $Z_{1} \cap Z_{2}$ is a Whitney stratified space, whose strata are obtained by intersecting the strata of $Z_{1}$ and $Z_{2}$. Let $p \in Z_{1} \cap Z_{2}$ be a point, and let $S_{1} \subseteq Z_{1}$ and $S_{2} \subseteq Z_{2}$ be the two strata containing it. Let $S_{1} \cap S_{2}$ be the corresponding stratum of $Z_{1} \cap Z_{2}$ containing $p$. If $Q_{1} \subseteq R^{d}$ is a $d_{1}$-simplex spanned by $d_{1}$ unit vectors and $Q_{2} \subseteq R^{d}$ is a $d_{2}$-simplex spanned by $d_{2}$ unit vectors, that are independent of the above set of $d_{1}$ vectors, then we let $Q_{1} \otimes Q_{2}$ denote the $\left(d_{1}+d_{2}\right)$-simplex spanned by the combined collection of $d_{1}+d_{2}$ unit vectors.

Now let $N_{1}(p)$ and $N_{2}(p)$ denote the normal slices of $Z_{1}$ and $Z_{2}$ at $p$. Let $N(p)$ denote the normal slice of $Z_{1} \cap Z_{2}$ at $p$. Then because of transversality it is clear that the stratification $N(p)$ coincides with that of $N_{1}(p) \otimes N_{2}(p)$, up to homeomorphism. Moreover,

$$
\begin{aligned}
\operatorname{dim}(N(p)) & =\operatorname{dim}\left(N_{1}(p)\right)+\operatorname{dim}\left(N_{2}(p)\right) \\
& =\operatorname{dim}\left(Z_{1}\right)-\operatorname{dim}\left(S_{1}\right)+\operatorname{dim}\left(Z_{2}\right)-\operatorname{dim}\left(S_{2}\right) \\
& =\operatorname{dim}(Z)-\operatorname{dim}(S),
\end{aligned}
$$

where the last equality holds because $Z_{1}$ and $Z_{2}$ are transversal.

Corollary 3.2. If $M_{1}, \ldots, M_{n}$ are smooth manifolds with boundaries, that intersect transversally, then $\bigcap_{i=1}^{n} M_{i}$ is a simple, stratified space.

Proof. We have already seen that each manifold $M_{i}$ has a simple stratification $\left\{M_{i} \backslash \partial M_{i}, \partial M_{i}\right\}$. Now apply Theorem 3.2 .

Another useful property of simple stratified sets is the following.

Lemma 3.1. Let $Z$ be a simple, stratified set. Let $S$ be a stratum of $Z$ and let $\bar{S}$ denote its closure. Then $\bar{S}$ is a simple, stratified set, whose stratification consists of the strata of $Z$ contained in $\bar{S}$.

Proof. Obvious.

Now let $Z$ be any compact, simple space. If $S$ is a stratum of $Z$, let $\chi(S)$ denote its Euler's characteristic. Let $f^{i}(Z)=\sum_{s} \chi(S)$, where $S$ ranges over all $i$-dimensional strata of $Z$. 
Theorem 3.3. For $0 \leq i \leq d=\operatorname{dim}(Z)$,

$$
\sum_{j=0}^{i}(-1)^{j}\left(\begin{array}{l}
d-j \\
d-i
\end{array}\right) f^{j}(Z)=f^{i}(Z) .
$$

Proof. First we prove that, for each stratum $S$ of $Z$,

$$
\sum_{R \subseteq S}(-1)^{\operatorname{dim}(R)} \chi(R)=\chi(S)
$$

Here $\bar{S}$ denotes the closure of $S$, and $R$ ranges over all strata of $Z$, contained in $\bar{S}$.

Consider a fixed stratum $R \subseteq \bar{S}$, and fix a field $\mathbf{F}$. Let $\partial R$ denote the boundary of $R$. If $R$ is not orientable, we let $\mathbf{F}=\mathbf{F}_{2}$. By Lefschetz duality,

$$
\beta_{i}(R ; \mathbf{F})=\beta_{\mathrm{dim}(R)-i}(\bar{R}, \partial R ; \mathbf{F})
$$

Here $\beta_{i}(R ; \mathbf{F})$ denotes the $i$ th Betti number of $R$ over $\mathbf{F}$, and $\beta_{i}(\bar{R}, \partial R ; \mathbf{F})$ denotes the $i$ th relative Betti number of the pair $(\bar{R}, \partial R)$. This immediately implies that

$$
\chi(R)=(-1)^{\operatorname{dim}(R)} \chi(\bar{R}, \partial R),
$$

where $\chi(\bar{R}, \partial R)$ denotes the relative Euler's characteristic of the pair $(\bar{R}, \partial R)$. Note that Euler's characteristic does not depend on the field $F$.

Using (5), equation (4) can be rewritten as

$$
\sum_{\mathbf{R} \subseteq S} \chi(\bar{R}, \partial R)=\chi(S)
$$

Let $S_{i}$ denote the union of the strata within $\bar{S}$, having dimension $\leq i$. Then applying the Excision Theorem for homology, and additivity of Euler's characteristic we get

$$
\sum_{\operatorname{dim}(R)=i} \chi(\bar{R}, \partial R)=\chi\left(S_{i}, S_{i-1}\right)
$$

Using (7), and the additivity of Euler's characteristic, the left-hand side of (6) becomes

$$
\sum_{R \leq S} \chi(\bar{R}, \partial R)=\sum_{i=0}^{\operatorname{dim}(S)} \chi\left(S_{i}, S_{i-1}\right)=\chi(\bar{S}) .
$$

Here, the last equality is obtained by repeatedly applying the identity

$$
\chi\left(S_{i}, S_{i-1}\right)+\chi\left(S_{i-1}\right)=\chi\left(S_{i}\right)
$$

Hence to prove (4), it remains to prove that $\chi(S)=\chi(\bar{S})$. However, notice that $\bar{S}$ is a topological manifold, i.e., every point $p$ in $\bar{S}$ has a closed neighborhood that is 
homeomorphic to a closed ball of dimension $\operatorname{dim}(S)$ : This is obvious if $p \in S$. If $p \in \partial S$, then this follows because $Z$ is simple, and hence $\bar{S}$ is simple, by Lemma 3.1.

Because $\bar{S}$ is a topological manifold, it follows from Brown's theorem [1] that $\partial S$ has a product neighborhood in $\bar{S}$, that is homeomorphic to $\partial S \times[0,1]$. This immediately implies that $S$ and $\bar{S}$ have the same homotopy type and hence $\chi(S)=\chi(\bar{S})$. This proves $(4)$.

Because Euler's characteristic is additive, we can henceforth assume in our proof, that each stratum is connected. This implies that the topological type of the normal slice $N(p)$ at $p \in Z$ depends only on the stratum $S=S(p)$ containing $p$. This essentially follows from Thom's Isotopy Lemma; see [4]. This implies that there is a one-to-one correspondence between the $j$-dimensional strata of $Z$ adjacent to $S$, where $j \geq \operatorname{dim}(S)$, and the $(j-\operatorname{dim}(S))$-dimensional strata of $N(p)$ that are adjacent to $p$. However, because $Z$ is simple, the stratification of $N(p)$ is identical (up to homeomorphism) to the canonical stratification of a simplex of dimension $=\operatorname{dim}(Z)-\operatorname{dim}(S)=d-\operatorname{dim}(S)$. In particular, there are precisely $\left(\begin{array}{c}d-\operatorname{dim}(S) \\ d-j\end{array}\right) j$-dimensional strata adjacent to $S$, where $j \geq \operatorname{dim}(S)$.

After this the proof is analogous to the proof of the classical Dehn-Sommerville relations. For each $i \leq d$, we sum both sides of (4) over all strata $S$ in $Z$ of dimension $i$. On the right-hand side, we get $\sum \chi(S)=f^{i}(Z)$. Each $j$-dimensional stratum $R$ is adjacent to $\left(\begin{array}{c}d-\operatorname{dim}(R) \\ d-i\end{array}\right)$ strata of dimension of $i$, and hence occurs as many times in the left-hand side during the summation. This immediately implies that the left-hand side, after summation, becomes

$$
\sum_{\operatorname{dim}(S)=i} \sum_{R \leq S} \chi(R)(-1)^{\operatorname{dim}(R)}=\sum_{R}\left(\begin{array}{c}
d-\operatorname{dim}(R) \\
d-i
\end{array}\right) \chi(R)
$$

where $R$ ranges over all strata in $Z$ of dimension $\leq i$.

Hence,

$$
\begin{aligned}
\text { left-hand side } & =\sum_{j}\left(\begin{array}{l}
d-j \\
d-i
\end{array}\right) \sum_{\mathrm{dim}(R)=j} \chi(R) \\
& =\sum_{j}\left(\begin{array}{l}
d-j \\
d-i
\end{array}\right) f^{j}(Z) .
\end{aligned}
$$

Hence,

$$
f^{i}(Z)=\sum_{j=0}^{i}\left(\begin{array}{l}
d-j \\
d-i
\end{array}\right) f^{j}(Z), \quad 0 \leq i \leq \operatorname{dim}(Z)
$$

Finally, notice that just like the classical Dehn-Sommerville relations admit a transparent reformation in terms of an $h$-vector, the relations in Theorem 3.3 too 
can be reformulated in an analgous fashion. If we let

$$
h^{i}(Z)=\sum_{j=0}^{d}(-1)^{i+j} f^{j}(Z), \quad 0 \leq i \leq d=\operatorname{dim}(Z),
$$

then Theorem 3.3 can be rewritten as follows.

Theorem 3.4. $h^{i}(Z)=h^{d-i}(Z), 0 \leq i \leq d=\operatorname{dim}(Z)$, for a simple, stratified set $Z$.

Remark. The strata of the simple spaces considered in this paper were smooth manifolds. However, the smoothness condition can be relaxed. Indeed, the results in this paper can be easily generalized to compact simple complexes whose building blocks are combinatorial manifolds [7].

\section{References}

1. M. Brown, Locally flat imbeddings of topological manifolds, Ann. of Math., 75 (1962), 331-341.

2. M. Dehn, Die Eulersche Formel in Zusammenhang mit dem Inhalt in der nicht-Euklidischen Geometrie, Math. Ann., 61 (1905), 561-586.

3. M. Golubitsky, V. Guillemin, Stable Mappings and Their Singularities, Springer-Verlag, New York, 1974.

4. M. Goresky, R. MacPherson, Stratified Morse Theory, Springer-Verlag, New York, 1987.

5. B. Grünbaum, Convex Polytopes, Wiley, New York, 1967.

6. V. Klee, A combinatorial analogue of Poincare's duality theorem, Canad. J. Math., 16 (1964), 517-531.

7. S. Lefschetz, Algebraic Topology, American Mathematical Society, Providence, RI, 1942.

8. P. MacMullen, G. Shephard, Convex Polytopes and the Upper Bound Conjecture, London Mathematical Society Lecture Notes Series 3, Cambridge University Press, Cambridge, 1971.

9. D. Sommerville, The relations connecting the angle sums and volume of a polytope in space of $n$ dimensions, Proc. Roy. Soc. London Ser. A, 115 (1927), 103-119.

10. R. Stanley, The number of faces of a simplicial convex polytope, Adv. in Math., 35 (1980), 236-238.

Received April 10, 1991. 